\section{Washing by website}

A new website that provides clinical staff (including dental and primary care) with guidance on the effective decontamination of surgical instruments has been introduced by specialist washing

company Dawmed International.

The site, www.dawmed-

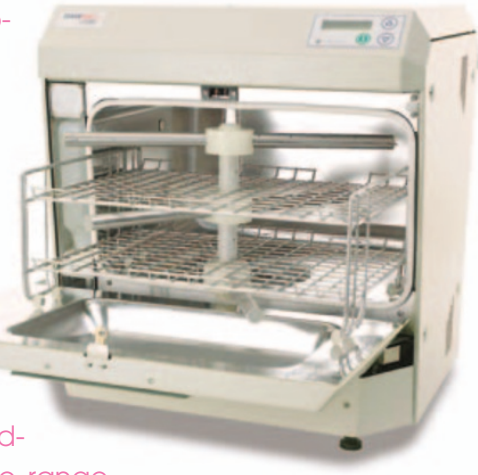

clinic.co.uk, provides a wide range

of news and information, covering key topics such as regulatory information, best practice, frequently asked questions and an innovative cost/feature comparisons tool to determine potential cost savings when moving from manual washing to an automated process

Within the website users can also see Dawmed's own solutions for the washing, disinfecting and drying of surgical instruments in the clinical environment, one of which is the new Dawmed clinic, a purpose designed compact washer disinfector dryer for applications where speed is essential and space is at a premium.

\section{Sterilise your mouse}

A computer mouse that can be completely sterilised simply by washing in antibacterial or sterilising fluid has been launched by Unotron Limite.

It is hoped that the ScrollSeal washable mouse may help to combat MRSA within clinical environments. The washable mouse complements the company's SpillSeal keyboard which uses patented technology to individually seal each key, therefore protecting the inter-

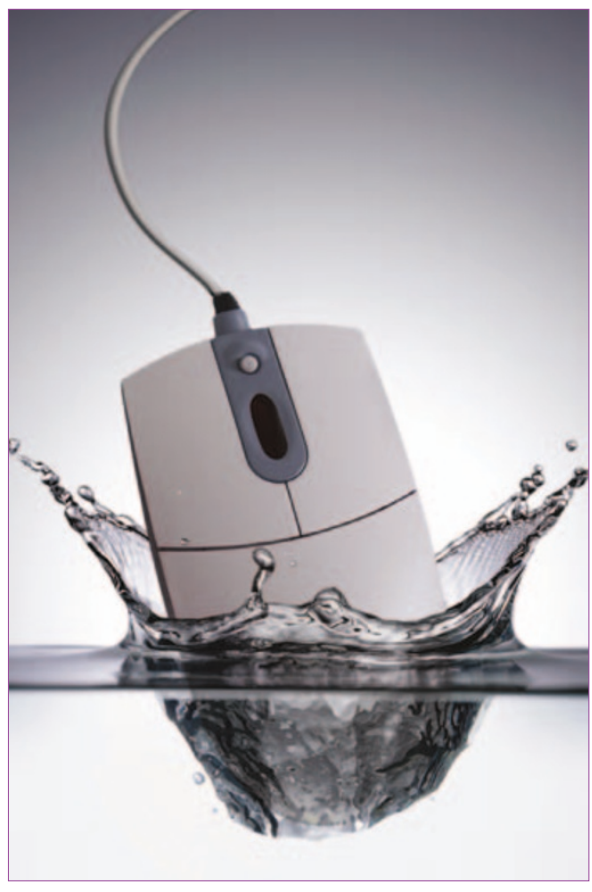

nal parts of the keyboard from liquid and dust while retaining the touch type feel of a top quality PC keyboard.

The affordable SpillSeal keyboards have widespread applications not only for dental clinics but also general hospital wards and clinics and other workplaces such as schools, offices and libraries. For more information visit www.unotrononline.co.uk.

\section{marketplace}

\section{Treating sensitive teeth}

Blackwell Supplies has introduced its latest product, Denshield, designed to reduce sensitivity and treat the cause of sensitive teeth. Denshield provides rapid and continuous relief and actively reduces open dentine tubules to prevent the exposure of the pulp nerve to stimuli. Available as a home-treatment dentifrice, there is also an optional in-office treatment that offers immediate short-term relief.

Denshield contains NovaMin (calcium sodium phosphosilicate), composed of elements all found naturally in the body which are safe and biocompatible, according to the company. These elements create natural conditions for tooth mineralisation, repairing surface lesion and reducing sensitivity and gingivitis. The new tooth mineral built with Denshield is real hydroxyapatite and actively protects the tooth nerve instead of just stopping the pain signal.

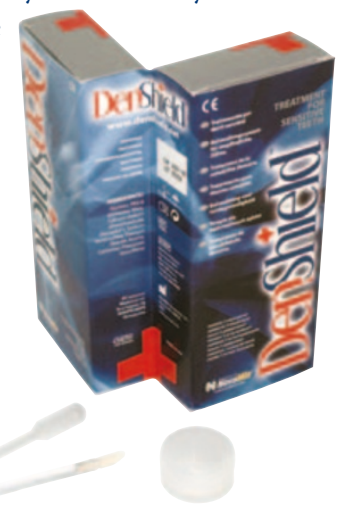

\section{Adept adhesion}

Futurabond NR SingleDose is specially designed to simplify safe adhesion. The newly-developed SingleDose packaging enables easy application - a comfortable route to safe adhesion.

Futurabond NR SingleDose is reinforced with nano particles and achieves outstanding shear bond strength on dentine and enamel. The micro-tensile bond strength of the product on dentine is $35.6 \mathrm{MPa}$ and combined with the nano-hybrid composite Grandio, shows excellent marginal tightness even under extreme loads.

The new SingleDose blister facilitates fast and optimal mixing: simply press and then open with a micro-applicator. Since Futurabond NR only has to be applied in one layer, the bonding step is reduced to a couple of seconds and is very tolerant to varying degrees of moisture on dentine. Available from wWW.voco.com.

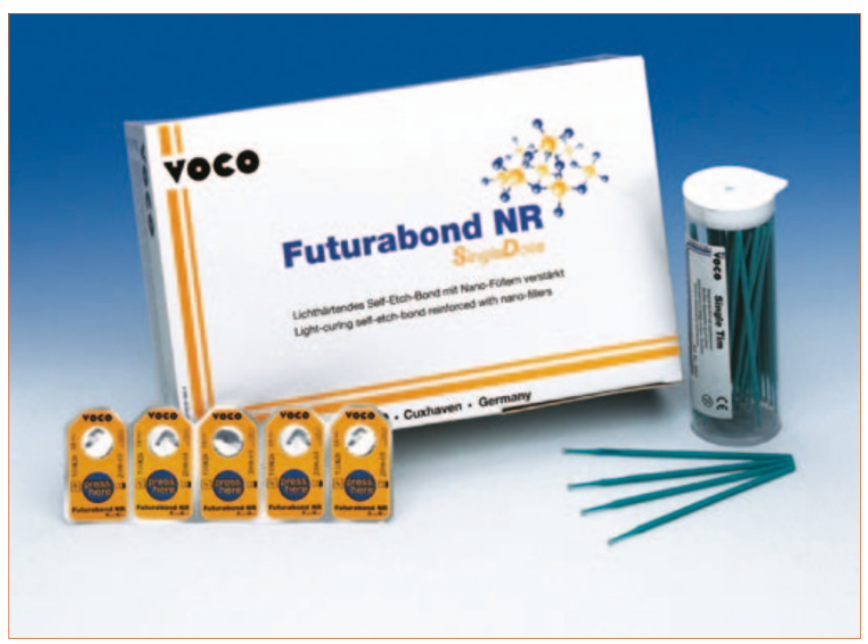

